\title{
De Leipzig à Fès : une copie sépharade de la traduction hébraïque du Coran d'Hermann Reckendorf
}

Par

Naïma Afif

Université de Manchester, Université catholique de Louvain

A

u dix-neuvième siècle est réalisée la première traduction du Coran en hébreu faite à partir de l'arabe. Produite par l'orientaliste allemand Hermann Reckendorf (1825-

1875 ) pendant les Lumières juives, cette version coranique (désormais abrégée en Der Koran) est rédigée principalement en hébreu biblique et caractérisée par un phénomène de judaïsation ${ }^{1}$. Le catalogue de la Bibliothèque Nationale d'Israël indique qu'une partie de la traduction est préservée dans un manuscrit sépharade datant de la même période (Jérusalem, Bibliothèque Nationale d'Israël, Heb. $\left.8^{\circ} 6453\right)^{2}$. Originaire du Maroc et copié durant la seconde moitié du $19^{\mathrm{e}}$ siècle sur base d'une édition imprimée, le document a appartenu à Aharon Botbol (1867-1946), membre du premier Tribunal Rabbinique de Fès ${ }^{3}$.

Durant le dernier quart du $19^{\mathrm{e}}$ siècle, le mouvement idéologique de la Haskala connaît en effet une diffusion en Afrique du Nord - dont le Maroc - dans quelques cercles privilégiés. Ces

1 Afif 2015. Au sujet de la traduction de Reckendorf et des traductions ultérieures du Coran en hébreu, voir Afif 2012 et Harif 2019.

${ }^{2}$ Une reproduction digitale du manuscrit accompagnée d'une brève description est disponible en libre accès sur le site du catalogue de la Bibliothèque Nationale d'Israël : http://web.nli.org.il/sites/NLI/Hebrew/digitallibrary/pages/viewer.aspx?presentorid=MANUSCRIPTS\&docid=P NX_MANUSCRIPTS000040814-1\#|FL16475897

${ }^{3}$ Voir Protectorat 1918, p. 990. 
contacts se manifestent notamment à travers la circulation de nouvelles œuvres ${ }^{4}$. La copie fassie de la traduction de Reckendorf, publiée à Leipzig, en témoigne à juste titre et offre une occasion inédite d'étudier le transfert du Coran en hébreu en Afrique du Nord. Elle atteste en outre l'intérêt d'une élite religieuse judéo-marocaine pour le Coran en hébreu dans un pays musulman où l'arabe est pourtant connu des communautés juives locales ${ }^{5}$.

Après une description d'ensemble du manuscrit, un intérêt particulier sera accordé aux contenus paratextuels accompagnant la copie fassie de Der Koran. Ce matériel concerne principalement les notes personnelles du rabbin Botbol. Seront ainsi examinés pour la première fois les indices susceptibles d'éclairer la transmission et la réception de la traduction coranique de Reckendorf en milieu sépharade.

\section{Description du manuscrit}

Pendant longtemps, la communauté scientifique a considéré que Der Koran avait été publié en $1857^{6}$. Une recherche approfondie nous a cependant permis d'établir avec exactitude que la traduction était parue entre 1855 et 1857 en cinq livraisons formant quatre volumes de taille inégale ${ }^{7}$.

Le manuscrit Jérusalem, Bibliothèque nationale d'Israël , Heb. $8^{\circ} 6453$ contient l'équivalent de la troisième livraison de la traduction de Reckendorf éditée en 1856 : le texte coranique est identique, de même que la mise en page, les notes infrapaginales et la numération des versets (voir figures 1 et 2). Toutefois, la copie ne contient ni le titre de l'ouvrage, ni le nom du traducteur.

En dépit de l'influence des idées de la Haskala dans les grands centres urbains juifs d'Afrique du Nord à la fin du $19^{\mathrm{e}}$ siècle, les livres demeurent onéreux et leur importation est loin d'être aisée. De plus, l'imprimerie hébraïque est quasi inexistante au Maroc jusqu'à l'apparition du Protectorat français ${ }^{8}$. Dans ces conditions, il n'est guère surprenant que la traduction d'Hermann Reckendorf ait pu faire l'objet d'une copie.

Le manuscrit n'est pas daté avec précision et les circonstances de sa production sont inconnues. Il se présente sous la forme d'un cahier au papier bruni composé de 5 folios non numérotés et de folios paginés en continu de 1 à $284^{9}$. L'écriture du corps du texte à l'encre foncée correspond à une semi-cursive sépharade tardive ${ }^{10}$, à l'exception de la sourate liminaire qui, comme les titres des sourates, est en caractères carrés (voir figure 3). L'identité du scribe est inconnue. Des notes de la main d'Aharon Botbol accompagnent la traduction ; elles sont en lettres cursives tracées tantôt à l'encre brune, tantôt à l'encre bleue (cf. chapitre suivant). Après un folio blanc, les pages numérotées de 1 à 240 contiennent le texte coranique avec de rares notes marginales. Les pages 241 à 247, 264 et 266 à 283 sont vierges. Un ex-dono indique que le document a été acquis par la Bibliothèque nationale d'Israël grâce au fonds établi par un

\footnotetext{
${ }^{4}$ Sur le sujet, voir CHÉTRIT 2006.

${ }^{5}$ ZAFRANI 1967.

${ }^{6}$ RUBIN 2007, p. 304 ; BOBZIN 2005, p. 350-351 ; FRÄNKEL et R. GOTTHEIL 1904, p. 557-560.

${ }^{7}$ AFIF 2015, p. 19-27.

${ }^{8}$ ZAFRANI 1969, p. 74 et 101.

${ }^{9}$ Le chiffre 1 a été ajouté par le rabbin Botbol (נומירו du français «numéro »). De 2 à 240 , les chiffres sont écrits à l'encre foncée par le copiste. La numération des pages suivantes tracée au crayon semble avoir été ajoutée ultérieurement.

${ }^{10}$ YARDENI 2002, p. 96-97.
} 
certain Ronald Marks (1940-1978) de Vancouver. Sur la dernière page, est tracé au crayon le nom de Jacob Abitbol (variante du patronyme Botbol), certainement un parent du rabbin de Fès qui a eu le manuscrit en sa possession.

\section{Les notes personnelles du propriétaire du manuscrit}

L'examen du paratexte du manuscrit présente un double intérêt. En effet, il éclaire à la fois l'histoire de la transmission et la réception de Der Koran en milieu sépharade. Dans les chapitres qui suivent seront analysées les notes du rabbin Botbol. Ces dernières comprennent : 1) la signature du rabbin et ses notes de lecture ;2) un commentaire portant sur l'identification du traducteur ; 3) la table des matières de la traduction ; 4) une transcription de la sourate une en arabe et en lettres hébraïques.

\section{Signature et notes de lecture du rabbin}

Les notes additionnelles contenues dans le manuscrit de Jérusalem sont de la main d'Aharon Botbol. Cette attribution est confirmée par une comparaison paléographique avec la signature du rabbin apposée au bas d'un acte officiel datant de 1945, la halitza ${ }^{11}$ de Simha fille d'Isaac Ha-Cohen qui est conservée au Centre de la Culture Judéo-Marocaine à Bruxelles (voir figure 4). De plus, à la première page du manuscrit de Jérusalem, le rabbin Botbol s'identifie au moyen d'un acronyme, comme le signale le catalogue des manuscrits de la Bibliothèque nationale d'Israël : אהב"א [=אהרן בכר אברהם בוטבול מפאס]2 קנין כספי לקיים ודע מה שתשיב וכו", הוה העבד אנכי («Acquisition confirmant sache ce que tu réponds etc. ; c'est la règle établie par la loi ; une parole agréable écrite et donnée [par] Aharon, le fils premier-né d'Abraham Botbol de Fès »). Le rabbin cite un extrait de la Mishna : "Sois prompt à étudier la Torah et sache ce que tu réponds à l'épicurien... » (Avot 2,14), le mot « épicurien » désignant ici l'impie ${ }^{13}$. En renvoyant à cette citation talmudique, Aharon Botbol explique l'intérêt de posséder une traduction coranique en hébreu : il s'agit d'avoir connaissance du livre sacré des musulmans afin d'être en mesure de répondre à d'éventuelles réfutations de leur part. L'homme de foi semble néanmoins davantage exprimer son intégrité religieuse plutôt qu'une disposition d'esprit polémique, si l'on tient compte de la nature objective de ses notes de lecture qui sont purement descriptives. Le rabbin se contente en effet de noter les références de versets coraniques classés thématiquement, en pointant au passage certaines similarités ou différences entre l'islam et le judaïsme. Ces notes couvrent les sujets suivants : une liste de noms propres d'origine arabe et hébraïque mentionnés dans le Coran (p. 249-254); les enfants d'Israël (p. 255-256); des lois coraniques concernant notamment l'interdiction de consommer du sang, du porc et de la nourriture sans avoir mentionné le nom divin, les femmes, le vin, l'obligation d'honorer son père et sa mère (p. 257-259); Mahomet (p. 260-261 et 265); la Kaaba (p. 262); des remarques diverses : les houris, le jour de la résurrection, la rétribution des œuvres, la géhenne, etc. (p. 263).

\footnotetext{
${ }^{11}$ Le rituel de la halitza, sanctionné par un document écrit, permet à la veuve de se libérer de l'obligation du lévirat.

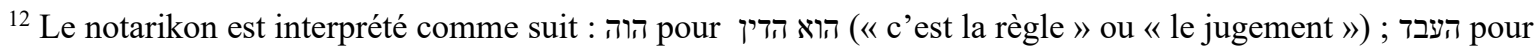

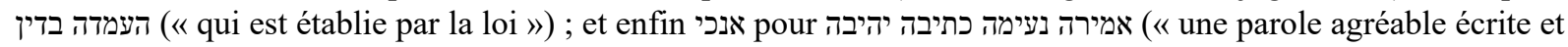
donnée ») ou אנא נפשי כתבית יהבית («j’ai moi-même écrit et donné »).

${ }^{13}$ Sur la signification et l'évolution du terme, voir LABENDZ 2003.
} 


\section{De l'identité du traducteur}

À la p. 248 du manuscrit, Aharon Botbol précise que seules quelques remarques et notes contenues dans la copie sont de sa main et qu'il n'est en aucun cas le traducteur du texte coranique. Une question se pose : le rabbin de Fès qui était en possession de cette copie de Der Koran connaissait-il l'identité de l'auteur de la traduction? Il semble que non. En effet, aux folios $1 \mathrm{r}-1 \mathrm{v}$, une glose écrite à l'encre brune, discute de la possibilité de relier la traduction contenue dans le manuscrit de Jérusalem à deux importantes autorités rabbiniques sépharades au Moyen-Age : Simon Duran (Rachbats) et Isaac Bar Chechet (Rivach). Le rabbin Botbol accorde une préférence au premier en raison, explique-t-il, de sa meilleure connaissance de la langue arabe. Dans un texte dirigé contre le christianisme et l'islam intitulé L'arc et le bouclier, Simon Duran a, de fait, joint à son exposé des citations tirées de la sunna ainsi que des extraits coraniques, mais il n'a pas produit de traduction complète du Coran ${ }^{14}$. Une autre note du rabbin, à l'encre bleue, attribue potentiellement la traduction contenue dans le manuscrit à Jacob Israël Ha-Levi $\left(17^{\mathrm{e}} \mathrm{s}\right.$.). Rabbin à Zante et ses environs, ce dernier séjourna à Venise où il réalisa en effet une traduction de l'intégralité du Coran en hébreu. Pour étayer son propos, le rabbin Botbol mentionne trois références qui donnent un aperçu de la diversité des sources juives anciennes et modernes qu'il a pu consulter :

- L'intelligence des temps d'Azaria Figo (16e s.), rabbin de Venise et disciple de Léon de Modène 15 ;

- Le nom des grands, dictionnaire bibliographique du décisionnaire sépharade Haïm Joseph David Azoulay (18e/19e s.) ;

- L'histoire des juifs de l'historien et maskil Heinrich Graetz (19e s.).

À partir de ces sources, Botbol déduit que la traduction de Ha-Levi est basée sur une traduction latine. Les études les plus récentes ont toutefois montré que cette version hébraïque avait été établie à partir de l'Alcorano d'Arrivabene, première traduction du Coran en italien ${ }^{16}$.

Les notes et les suppositions avancées par le rabbin de Fès indiquent bel et bien que ce dernier ignorait l'identité de l'auteur de la traduction coranique contenue dans le manuscrit qu'il avait en sa possession.

\section{La table des matières}

Après cette discussion sur l'identité du traducteur, Aharon Botbol a inséré une table des matières. Les folios $2 \mathrm{v}-4 \mathrm{v}$ contiennent les références et les noms des sourates 1 à 30 en hébreu ${ }^{17}$. Ces derniers sont traduits en arabe écrit en caractères hébraïques (voir figure 5). L'usage de l'alphabet hébreu pour transcrire la langue arabe n'est pas exceptionnel dans les milieux juifs du Maghreb et d'Orient. Il n'y a donc rien d'étonnant à ce que le rabbin Botbol y ait eu recours.

\footnotetext{
${ }^{14}$ La section concernant l'islam a été éditée par M. Steinschneider qui en a également livré une traduction en allemand durant la dernière phase de la Haskala (seconde moitié du $19^{\mathrm{e}}$ siècle), période à laquelle est publiée la traduction du Coran en hébreu de Reckendorf, cf. STEINSCHNEIDER 1880. Pour l'édition du texte hébraïque, STEINSCHNEIDER 1881.

${ }^{15}$ Léon de Modène est l'auteur d'une notice de cinq pages contenant des informations sur l'islam en italien et en hébreu. Il se serait basé sur l'édition du Coran en italien publiée par Arrivabene. A ce propos, voir ADELMAN 2012. L'édition d'Arrivabene a servi de texte-source à la première traduction intégrale du Coran en hébreu, voir note suivante.

16 Voir LAZARUS-YAFEH 1998/1999 ; BASAL 2011a et 2011b.

${ }^{17}$ Notons que sur la copie digitale du manuscrit disponible en ligne, ces folios apparaissent en premier et ne sont pas à leur place.
} 


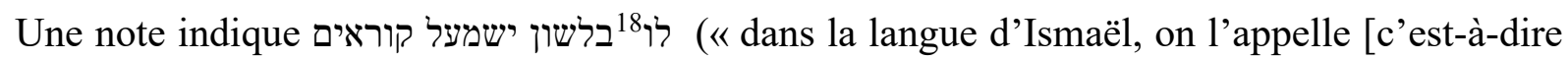
chacune des sourates] »). Cette liste de mots en langue arabe, qui ne figure pas dans le texte original de Der Koran, indique qu'Aharon Botbol a certainement eu accès au texte de la Vulgate coranique. Vient ensuite la suite de la table des matières de la traduction de Reckendorf (sourates 31 à 114) dans laquelle les noms sont donnés en hébreu uniquement. Le système de pagination correspond à celui de l'édition définitive de Der Koran (1857). Un autre manuscrit devait donc contenir le reste de la traduction du Coran de l'orientaliste allemand. Bien qu'il n'en existe aucune trace, cette possibilité est renforcée par une note du rabbin en haut de la première page du manuscrit signalant que le volume correspond à la «partie une » de la traduction (חלק א'). Une autre note au folio 3r indique que les sourates 31 à 114 se trouvent dans une deuxième partie (בחלק ב'). Il y a donc tout lieu de penser que la traduction du Coran de Reckendorf est parvenue au Maroc dans son intégralité.

\section{Translittération de la sourate 1}

Au bas de la première page numérotée du manuscrit, la sourate liminaire est écrite en arabe et en lettres hébraïques (voir figure 1). Il existe certes des translittérations du Coran en alphabet hébreu dont certaines ont été réalisées en milieu musulman ${ }^{19}$, mais l'échantillon écrit de la main du rabbin Botbol concerne un rare cas d'allographie du Coran produit au Maroc.

Cette translittération qui est destinée à un usage personnel ne présente toutefois pas un système homogène. Ainsi, un point suscrit sur le $p$ sert toujours à indiquer la vélaire occlusive sourde ك. La laryngale $\tau$ qui n'est pas réalisée en hébreu est rendue par la spirante fricative sourde $\pi$. En revanche, le vocalisme est tantôt indiqué par les matres lectionis, tantôt absent. Enfin, la gémination est rendue par une répétition de la lettre concernée ou complètement omise.

Voici le texte en question. Il est présenté en synopse avec la traduction de Reckendorf et le texte reçu de la sourate qui sont donnés à titre comparatif.

\begin{tabular}{|c|c|c|}
\hline 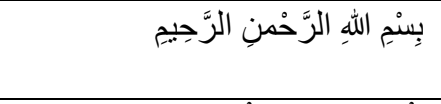 & בשם אלה הרחמן והרחום & ארראחים. \\
\hline الْحَمْدُ للّهِ رَبِّ الْعَالَمِيَنَ & (א) השבח לאלה רב העולמים. & נחמאלו ללאה רבב \\
\hline الرَّحْمَنِ الرَّحِيِِ & (ב) הרחמן והרחום. & . ראחמאני ראחים 21 . \\
\hline مَالِلِكِ يَوْجِ الآِيَّنِ & | (ג) מלך ביום הדין. & מאליקי יום דדין. \\
\hline إِيَّالكَ نَعْبُدُ و إِيَّاكَكَ نَسْتَعِعِينُ & |ד (ד) אותך נעבד ואליך נשוע. & אייאקא נעבודו וואייקה נאאסתאעיין. \\
\hline 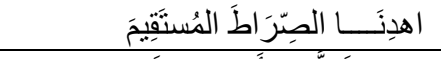 & (ה) הורנו בדרך המישרים ארחותיהם. & ייהדינא ללסירט אלאמוסתאקיםה. \\
\hline 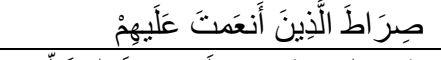 & (ו) דרך האנשים אשר חנות אותם. & סיראט אלאדינה נעמתא עאליהים. \\
\hline 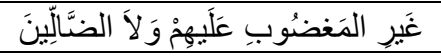 & (ז) לא דרך מכעיסיך ולא דרך התועים. & גאיירי למגדובי עאליהים וואדדאללין. \\
\hline
\end{tabular}

Une simple lecture du tableau synoptique montre que la translittération fournie par le rabbin Botbol ne consiste pas en une rétroversion de la sourate 1 faite à partir de l'hébreu. En effet, la

\footnotetext{
${ }^{18}$ La forme, orthographiée קורים dans le manuscrit, a été corrigée. Elle apparaît aussi avec un nun final, comme en hébreu rabbinique.

${ }^{19}$ Voir PAUDICE 2008.

${ }^{20}$ Le rabbin utilise ici la formule « en langue arabe ».

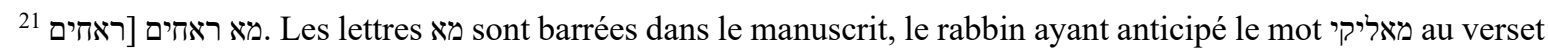
suivant.
} 
traduction de Reckendorf offre des particularités notables par rapport au texte reçu du Coran. A l'instar de la majorité des textes rédigés en hébreu pendant les Lumières juives, elle se caractérise par une propension à l'usage de l'hébreu biblique ainsi qu'au recours au shibbutz ${ }^{22}$

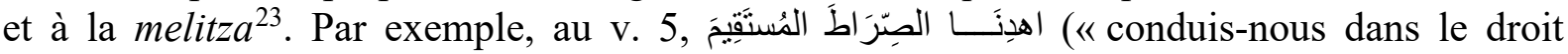
chemin ») devient הורנו בדרך המישרים ארחותיהם ("montre-nous la voie de ceux qui vont droit dans leurs chemins [= dans une voie droite]»). L'expression המישרים ארחותיהם renvoie à Prv

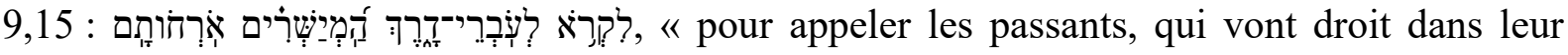
chemin » ou « dans une voie droite ».

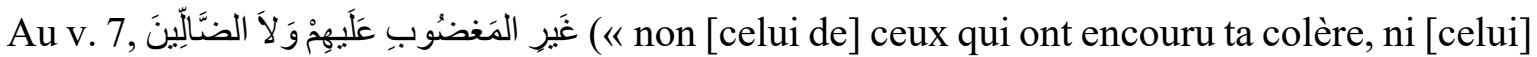
des égarés ») est traduit par לא דרך מכעיסיך ולא דרך התועים "non la voie de ceux qui t'ont irrité, ni la voie des égarés »). Il est fait mention de « ceux qui irritent » l'Eternel en hébreu, selon une expression biblique que l'on retrouve dans $1 \mathrm{R}$ 16,33 et 2 R 23,19. Reckendorf répète en outre le terme דרך (« voie »). Il offre un parallélisme antithétique qui montre un contraste entre l'idée de voie « droite » mentionnée au verset 6 de la sourate. Antithèses et parallélismes constituent des traits caractéristiques de la poésie biblique, particulièrement dans les Proverbes. Reckendorf privilégie ainsi la langue et la culture d'arrivée.

La translittération de la sourate une dans le manuscrit de Jérusalem en revanche est fidèle au texte de la Vulgate coranique à l'exception de quelques passages. Dans le premier verset exprimant une action de grâce, le segment « la louange est à Dieu » est rendu par un inaccompli de la $1^{\mathrm{e}}$ pers. plur. ayant une nuance exhortative et un complément d'objet נחמזו ללאה («ouons Dieu »).

ללסירט D'autres écarts semblent être imputables à des erreurs de graphie : par exemple

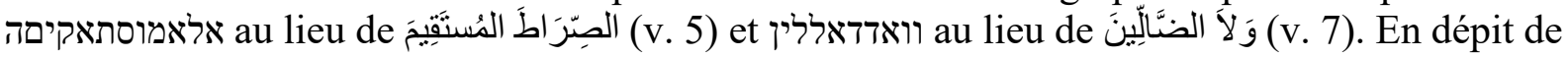
ces divergences mineures, le rabbin Bobtol a bel et bien restitué le texte reçu de la sourate 1 dans sa translittération.

\section{Conclusion}

Le manuscrit Jérusalem, Bibliothèque nationale d'Israël, Heb. $8^{\circ} 6453$ fournit les rares indices disponibles sur la transmission et la réception de la traduction du Coran en hébreu d'Hermann Reckendorf en milieu sépharade. Même si le copiste a pu avoir connaissance de l'identité du traducteur, il semble à la faveur des renseignements fournis par le document que la traduction coranique ait été transmise de façon anonyme. Par ailleurs, le contenu de la copie correspond à celui de la troisième livraison de la traduction de Reckendorf datant de 1856. Doiton pour autant en déduire que le transfert de la traduction s'est opéré de manière partielle ? Rien n'est moins sûr. Le manuscrit présente la table des matières de l'édition intégrale de 1857 et évoque une seconde partie de la copie de la traduction dans les notes marginales. En dépit de l'absence de trace matérielle des sourates 31 à 114, il est donc plus que probable que la première version du Coran en hébreu à partir de l'arabe soit parvenue au Maroc dans son intégralité.

Le paratexte donne un aperçu des connaissances du rabbin sur le Coran en hébreu et en arabe. Afin d'identifier l'auteur de la traduction, le rabbin a consulté des sources juives anciennes et modernes. Bien qu'il n'y soit pas parvenu, il a pu retracer un pan de l'histoire du transfert du

\footnotetext{
${ }^{22}$ Le terme est dérivé de la racine שבץ (« tresser », « broder ») et désigne un procédé stylistique consistant à insérer des versets ou expressions bibliques dans le texte produit. Ce procédé remonte à l'époque médiévale.

${ }^{23}$ La melitza est une technique de composition littéraire qui consiste à recourir à des figures de style proches de celles de la Bible. Le terme revêt souvent une acception péjorative et désigne un style fleuri et grandiloquent, cf. PELLI 2010.
} 
Coran en hébreu puisqu'il mentionne l'œuvre polémique de Simon Duran et la traduction de Jacob Israël Ha-Levi.

L'examen du paratexte illustre également le point de vue du rabbin sur le texte coranique. Ses notes de lecture témoignent de son intérêt pour les rapports ainsi que les différences entre l'islam et le judaïsme. Enfin, la translittération de la liste des sourates 1 à 30 et celle de la sourate liminaire laissent penser qu'Aharon Botbol a certainement eu à sa disposition une version arabe du Coran, facilement accessible en milieu musulman à l'époque moderne.

La traduction de Reckendorf sous sa forme définitive sera supplantée par une version en hébreu moderne produite par le professeur J.J. Rivlin de l'Université hébraïque de Jérusalem dès la première moitié du vingtième siècle ${ }^{24}$. Il n'en demeure pas moins qu'à un moment de sa courte diffusion, et en dépit du rayonnement restreint de la Haskala en Afrique du Nord, la traduction de Reckendorf publiée à Leipzig a transité jusqu'au mellah de Fès, dépassant ainsi les frontières du monde ashkénaze pour rejoindre la bibliothèque d'une haute instance rabbinique de la communauté juive marocaine. 


\section{ILLUSTRATIONS}

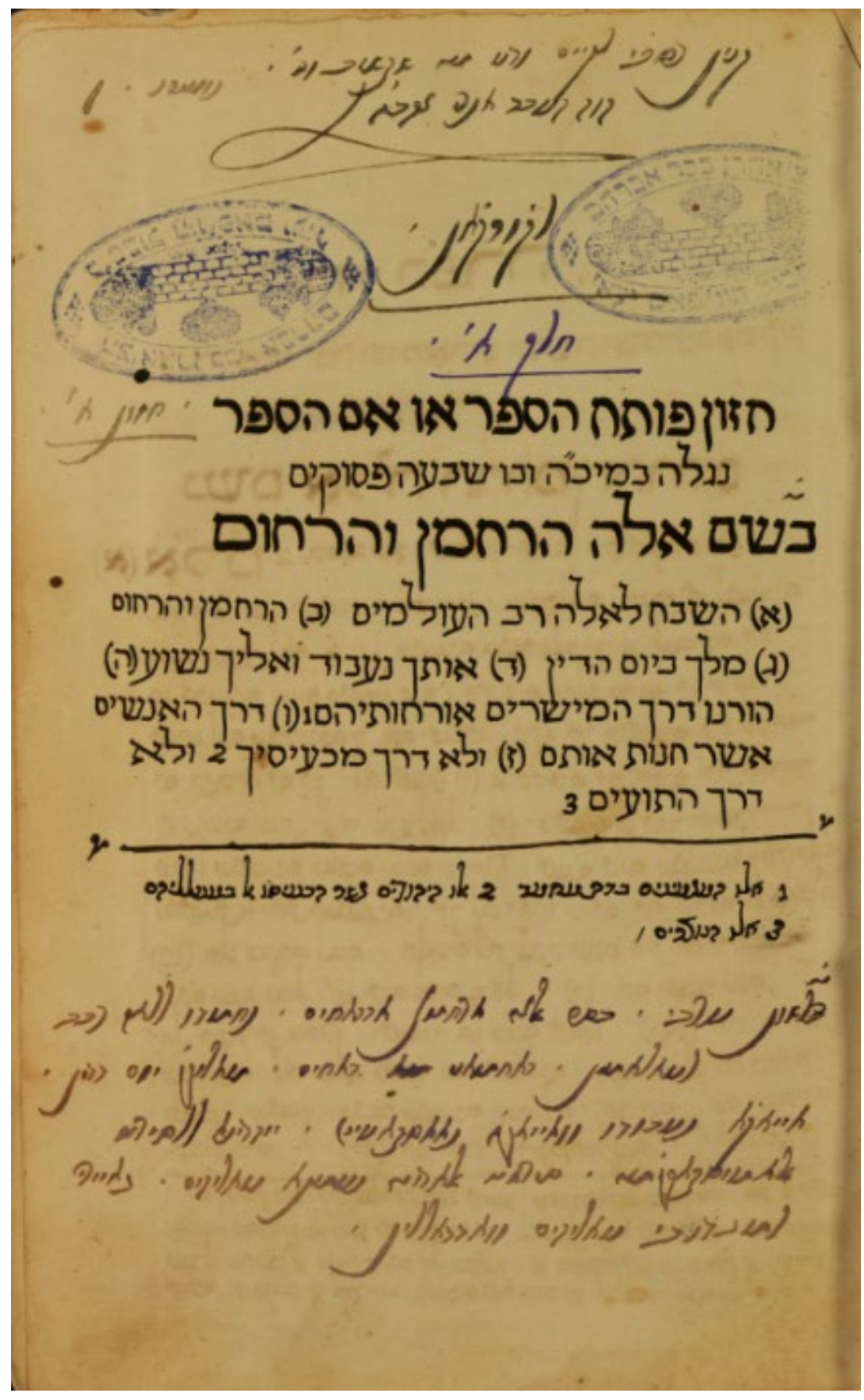

Fig. 1 : p. 1 du manuscrit Heb. Jerusalem $8^{\circ} 6453$.

Sourate une, notes infrapaginales et translittération de l'arabe en lettres hébräques. 


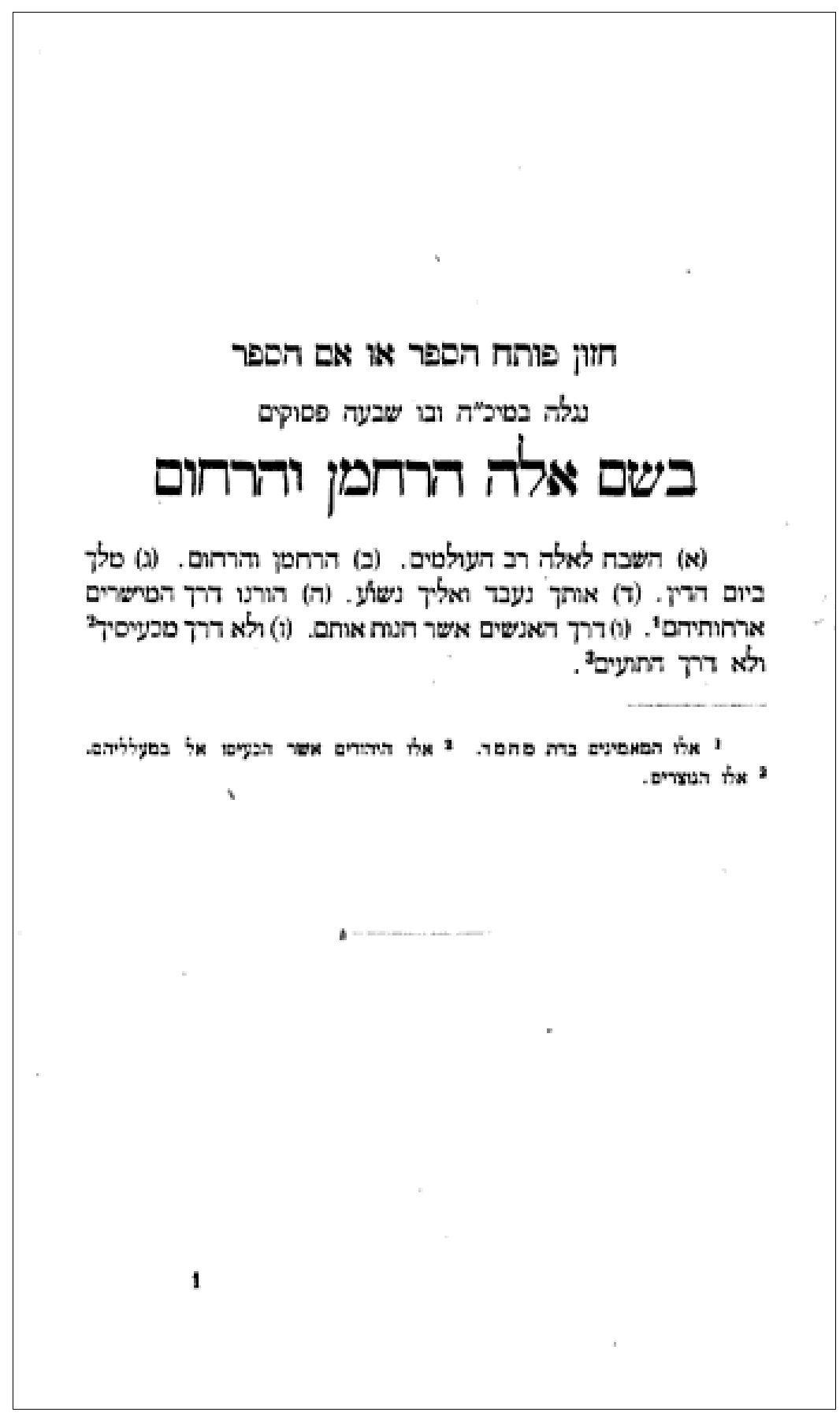

Fig. 2 : p. 1 de Der Koran (1856). Sourate une et notes infrapaginales. 


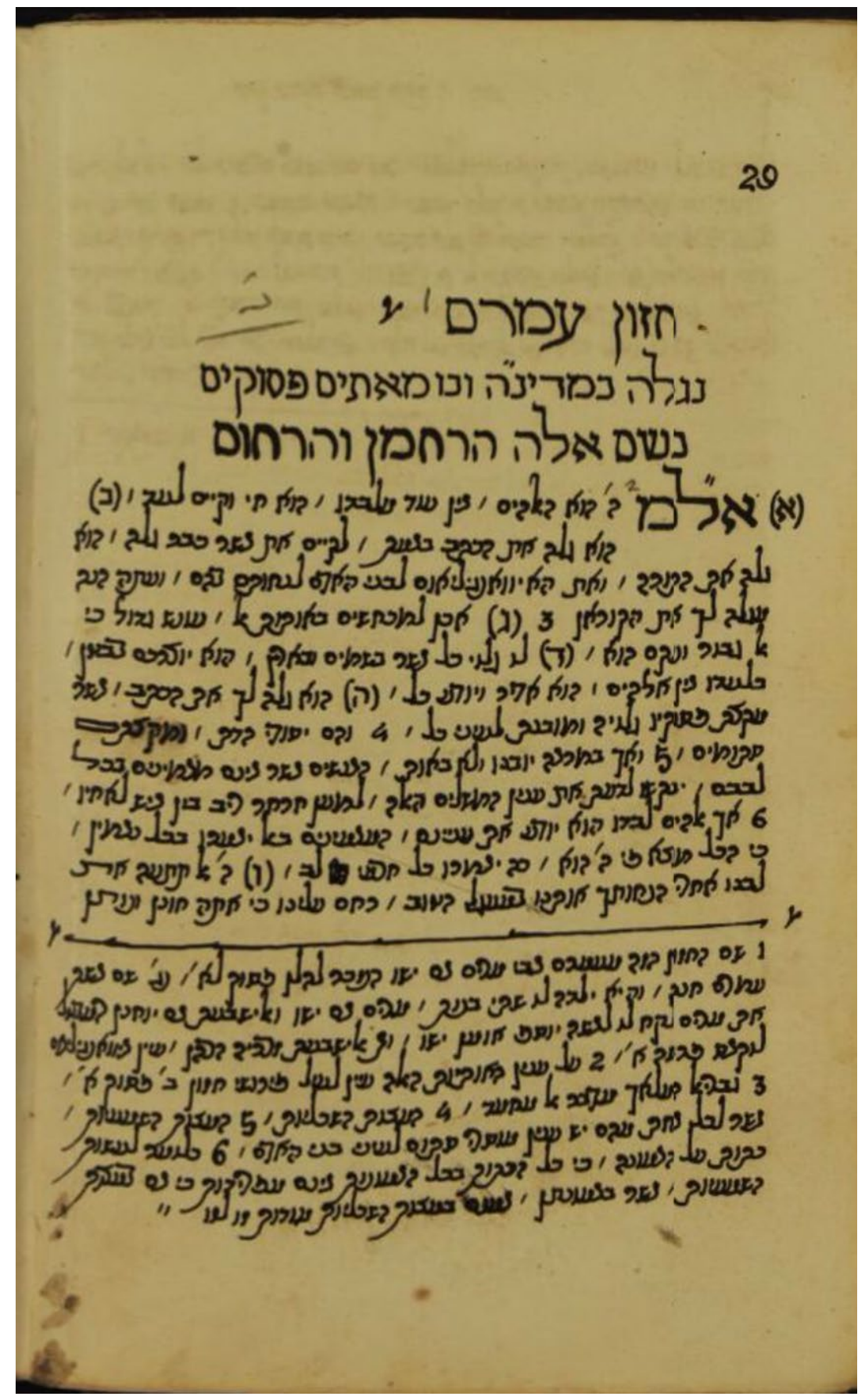

Fig. 3 : p. 29 du manuscrit Heb. Jerusalem $8^{\circ} 6453$. Début de la sourate 3. 


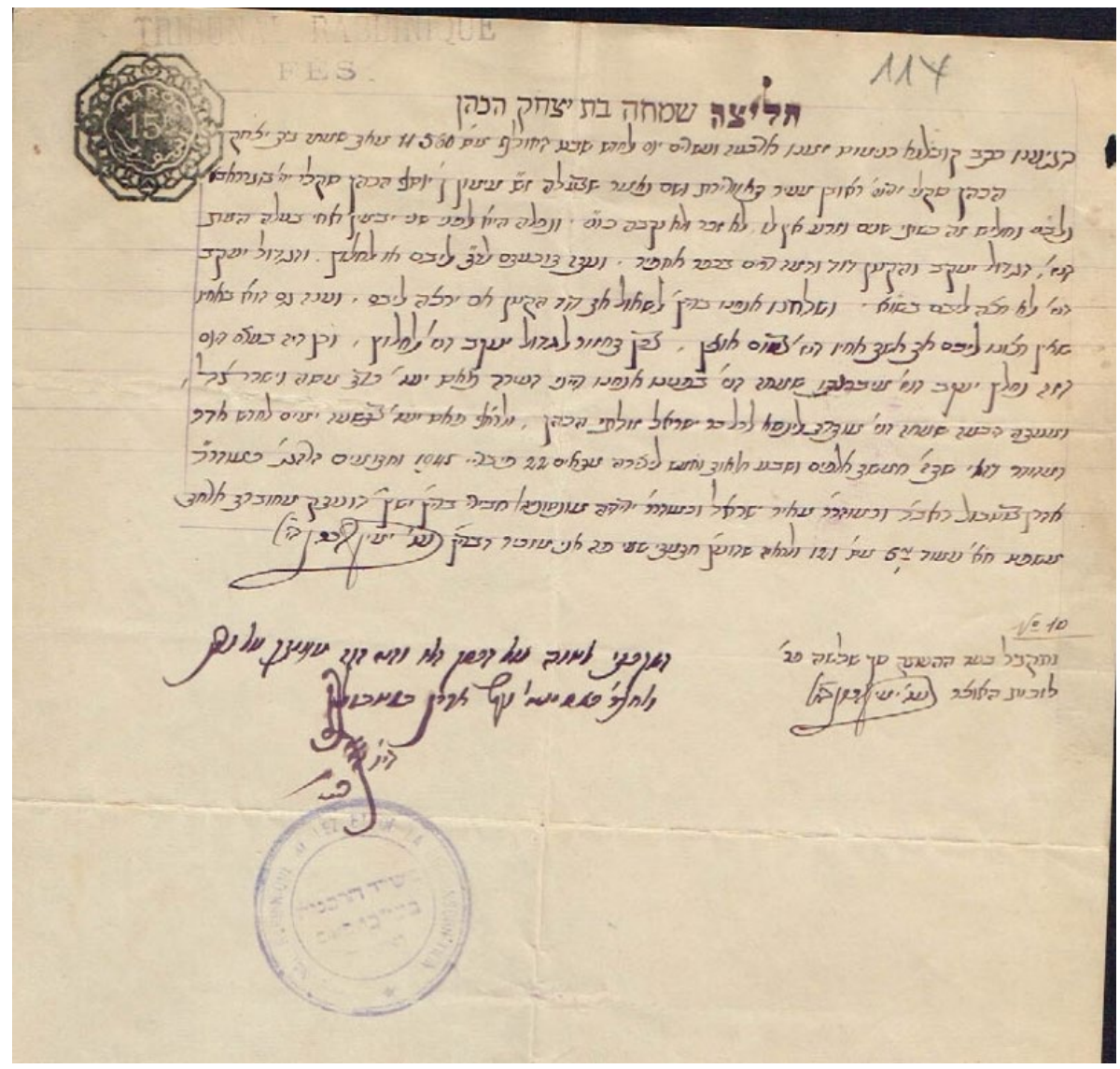

Fig. 4 : Halitza de Simha fille de Isaac Ha-Cohen (Centre de la Culture Judéo-Marocaine à Bruxelles, objet numéro 31074). L'écriture du rabbin Aharon Botbol figure au-dessus du cachet.

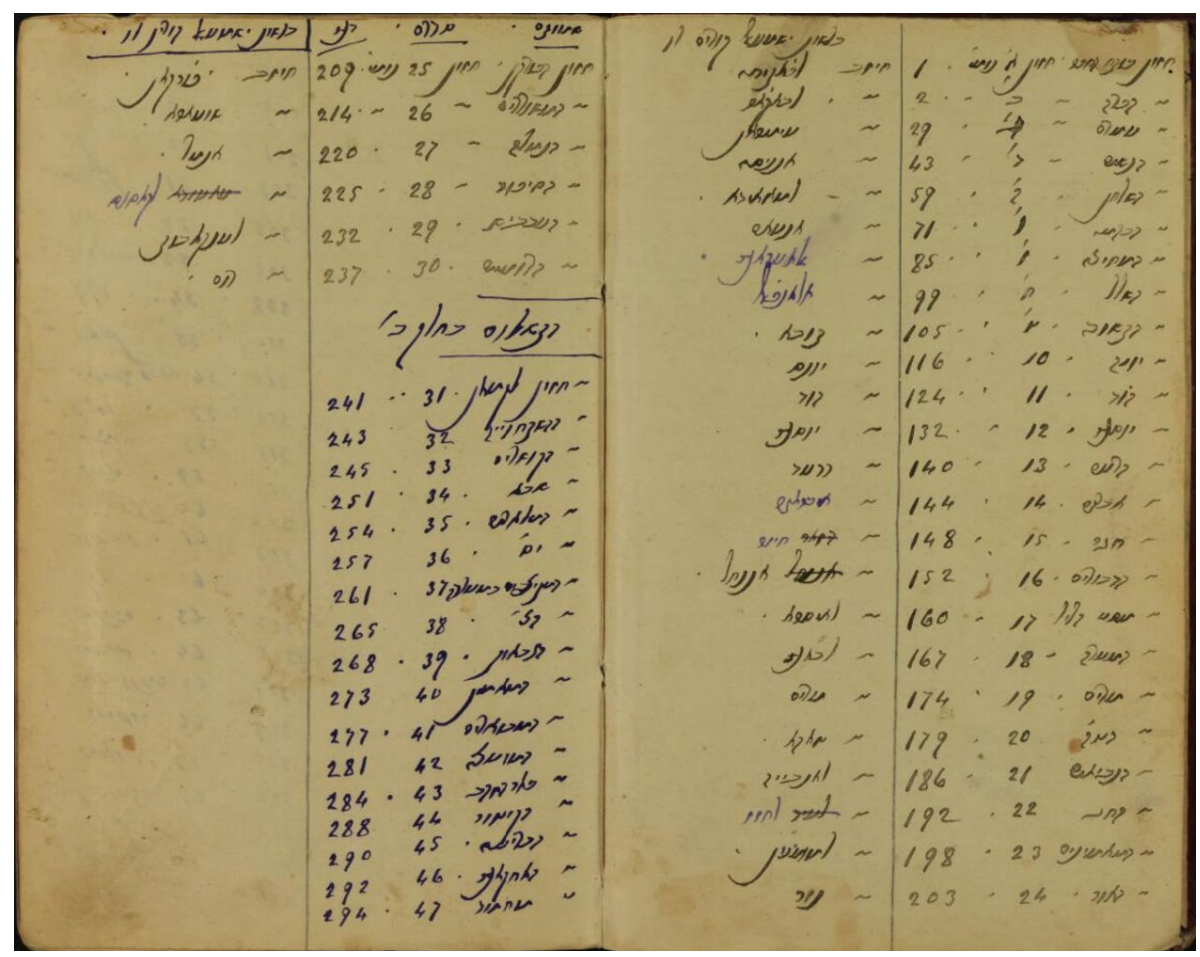

Fig. 5 : folios 2v-3r du manuscrit Heb. Jerusalem $8^{\circ} 6453$. Table des matières des sourates 1 à 47 . 


\section{BIBLIOGRAPHIE}

Adelman 2012 : H. T. Adelman, « A rabbi reads the Qur'an in the Venezian ghetto », Jewish History 26 1/2, p. 125-137.

AfIF 2012 : N. AfIF, «Les traductions contemporaines du Coran en hébreu », Acta Orientalia Belgica 25, p. 295-306.

- 2015 : N. AfIF, Une version biblique du Coran en hébreu. La traduction d'Hermann Reckendorf (1857). Modalités et étendue de la judaïsation, étude de la langue et réception pendant les Lumières juives, Thèse de doctorat en Langues et lettres, Université catholique de Louvain.

BASAl 2011a : N. BASAL, «A Seventeenth Hebrew Translation of the Qur'an and Its Arabic Sources" dans F. Musall et A. Al-MudarRis (éds), Im Dialog bleiben. Sprache und Denken in den Kulturen des Vorderen Orients (Festschrift für Raif Georges Khoury), Wiesbaden, p. 200-218.

— 2011b : «A propos d'une traduction du Coran en hébreu : la traduction de Jacob ben Israël Ha-Levi à partir de l'italien (Venise, 1636) », dans A. OETTINGER et D. BAR-MAOZ (éds), Mittuv Yosef : Yosef Tobi Jubilee Volume, vol. 3, Haïfa, p. 166-181 (en hébreu).

BobzIN 2005 : H. BobzIN, « Translations of the Qur'an », Encyclopaedia of the Qur'an, vol. 5, p. $350-351$.

ChÉTRIT 2006 : J. ChÉTRIT, «La Haskala hébraïque dans le monde sépharade », dans S. TrigAnO (éd.), Le monde sépharade. I. Histoire, Paris, p. 745-809.

FrÄNKEL et GotTheIL 1904 : S. FrÄNKEL et R. GotTheIL, « Koran », Jewish Encyclopedia, vol. 7, p. 557-560.

HARIF 2016 : H. HARIF, « Islam in Zion? Yosef Yo'el Rivlin's Translation of the Qur'an and Its Place Within the New Hebrew Culture », Naharaim 10/1, p. 39-55.

- 2019 : H. HARIF, « Between Sacred and Profane: Three Modern Hebrew Translators of the Qur'ān », dans D. M. SAlzER, C. GAFNI et H. HARIF (éds), Wissenschaft des Judentums Beyond Tradition. Jewish Scholarship on the Sacred Texts of Judaism, Christianity and Islam, Berlin/Boston, p. 149-164.

LABENDZ 2003 : J. R. LABENDZ, «"Know What to Answer the Epicurean” : A Diachronic Study of the 'Apiqoros in Rabbinic Literature », Hebrew Union College Annual 74, p. 175-214.

LAZARUS-YAFEH 1998/1999: H. LAZARUS-YAFEH, «A Seventeenth-century Hebrew Translation of the Qur'an », Scripta Mediterranea 19/20, p. 199-211.

PAudice 2008: A. PAudice, «On Three Extant Sources of the Qur'an Transcribed in Hebrew », European Journal of Jewish Studies 2/2, p. 213-257.

PELli 2010 : M. PELLI, « The Role of Melitzah in Early Haskalah Literature and its Reception at the End of the Period », dans ID., Haskalah and Beyond: The Reception of the Hebrew Enlightenment and the Emergence of Haskalah Judaism, Lanham Md, p. 135-160.

Protectorat 1918 : Protectorat DE LA RÉPUBLIQUe FRANÇAISE AU MAROC, Arrêté viziriel du 5 octobre 1918 (28 Hidja 1336) portant nomination des membres du Tribunal rabbinique de Fès. Bulletin officiel. Edition française hebdomadaire, $\mathrm{n}^{\circ} 314$ du 28 octobre 1918 (7e année).

RivLin 1936/1945 : J. J. RivLin, Le Coran : traduction de l'arabe, Tel Aviv, 2 vol. (en hébreu). 
Rubin 2007 : U. RuBIN, "Koran (Hebrew Translations of the Koran) », Encyclopaedia Judaica, vol. 12, p. 304.

STEINSCHNEIDER 1880 : M. STEINSCHNEIDER, «Islam und Judenthum. Kritik des Islam von Simon Duran (1423) », Magazin für die Wissenschaft des Judenthums 7, p. 1-48.

- 1881 : M. STEINSCHNEIDER, «Qeshet u-Magen», Ozar Tob, Hebräische Beilage zum Magazin für die Wissenschaft des Judenthums 8, p. 1-36.

YARdeni 2002 : A. YARDENI, The Book of Hebrew Script : History, Palaeography, Script Styles, Calligraphy \& Design, London.

ZAFRANI 1967 : H. ZAFRANI, «Langues juives du Maroc », Revue de l'Occident musulman et de la Méditerranée 4/4, p. 175-188.

- 1969 : H. ZAFRANi, Pédagogie juive en terre d'Islam. L'enseignement traditionnel de l'hébreu et du judaïsme au Maroc, Paris. 


\title{
RÉSUMÉ
}

La première traduction du Coran en hébreu établie à partir de l'arabe a été réalisée par Hermann Reckendorf (1825-1875) pendant les Lumières juives. En marge de sa diffusion dans le monde ashkénaze, la traduction apparaît dans un manuscrit copié au Maroc au $19^{\mathrm{e}}$ siècle. Cet article examine cet unique témoin de façon à apporter un éclairage sur la réception de la traduction de l'orientaliste allemand en milieu sépharade.

\begin{abstract}
The first Hebrew translation of the Qur'ān from Arabic was produced by Hermann Reckendorf (1825-1875) during the Jewish Enlightenment. Beyond its diffusion in the Ashkenazic world, the translation appears in a manuscript copied in Morocco in the 19th century. This paper examines this unique witness in order to shed light on the reception of the translation of the German orientalist in Sephardic milieu.
\end{abstract}

